\title{
Journal \\ of the \\ Optical Society of America
}

Devoted to all Branches of Optics

\begin{tabular}{llc}
\hline \hline Vol. V & MAY, 1921 & Number 3 \\
\hline
\end{tabular}

THE GLARIMETER

An Instrument for Measuring the Gloss of Paper

BY

L. R. INGERSOLI

Some years ago the writer developed a method ${ }^{1}$ and instrument for measurement of the gloss or degree of finish of a sheet of paper by means of its polarizing action on obliquely reflected light. The instrument-called a "glarimeter" since it measured the gloss in terms of the "glare" or specularly reflected light—was not placed at that time on the market, in spite of the fact that it seemed to supply a long-felt want for an exact means of gloss measurement. This was partly because of the difficulty of securing the necessary optical parts during the war time and partly because of some inherent defects.

Not long ago, however, it was found that if the optical system of the polarimetric part was replaced by a modified form of that used in the Pickering ${ }^{2}$ polarimeter, these defects would be removed and at the same time the sensibility and accuracy greatly increased. Furthermore the required optical parts-Wollaston double-image prism, nicol and lens-could be fairly readily obtained. A number of these glarimeters have been made up and have already proved their usefulness in various paper mills, and accordingly it seems worth while to describe the new form of the instrument.

${ }^{1}$ Electrical World, 63, p. 645 (Mar. 21, 1914). It is of interest to note that a German scientist, Dr. Karl Kieser, has recently (apparently independently) developed a similar instrument making use of the same principle. See Zeit, für Angew. Chemie, 32, 1, p. 357 (Nov. 11, 1919).

2 E. C. Pickering, Proc. Am. Acad. Arts and Sci. 9, 1 (1873) :21, 294 (1885). 
The general principle of the instrument, i.e. the polarization method of gloss measurement, has been already described, ${ }^{3}$ but may be briefly reviewed. Light from a source subtending a certain solid angle-in this case .038, i.e. a $44.5 \mathrm{~mm}$ diameter circular aperture at $203 \mathrm{~mm}$ distance-falls on the surface of paper at an angle of approximately 57.5 degrees. Part of this light is diffusely reflected and part specularly, the latter being shown by experiment to be (almost) completely plane polarized. The instrument accordingly measures gloss in terms of the fraction,. which is polarized, of the light entering the eyepiece from the paper, that is, in terms of the fraction of its brightness which is due to polarized light.

In the new optical system of the polarimeter the light passes in turn an adjustable slit, Wollaston prism, convex lens, and nicol rotating in graduated circle. The object of the lens is to throw the slit sharply in focus and to throw out of focus the paper surface so that its minute irregularities will not appear. It also allows the instrument to be shortened somewhat.

On looking in the eyepiece one sees the two images of the slit formed by the double-image prism. These are brought into focus by pulling in or out the tube carrying the slit, and the slit width is adjusted until they form a clear bi-field with sharp dividing line. The nicol is then rotated until they are of equal brightness. Then if $\mathrm{D}^{2}$ and $\mathrm{S}^{2}$ represent the intensities of the diffusely and specularly reflected light (so directed as to enter the eye) before entering the Wollaston, and if the latter is set so that the specularly reflected light is completely extinguished in the lower image, the brightness of the upper and lower images-before nicol is interposed-will be proportional to $\mathrm{D}^{2} / 2+\mathrm{S}^{2}$ and $\mathrm{D}^{2} / 2$, respectively. When they are viewed through the nicol and balanced by turning its principal plane through an angle $A$ from parallelism with that of the lower slit image, we have

$$
\left(D^{2} / 2+\mathrm{S}^{2}\right) \sin ^{2} \mathrm{~A}=\mathrm{D}^{2} / 2 \cos ^{2} \mathrm{~A}
$$

from which we readily obtain

$$
\frac{\mathrm{S}^{2}}{\mathrm{D}^{2}+\mathrm{S}^{2}}=\cos 2 \mathrm{~A}
$$

${ }^{3}$ Electrical World, loc. cit. 
or the percentage polarization is given at once in terms of the cosine of a double angle.

The gloss may be expressed directly in terms of this per cent of polarization, if desired, and indeed it was formerly recommended that this be done. It is a mistake, however, to imagine that this furnishes a scale of measurement which is absolute, ${ }^{4}$ since it will vary with the angle subtended by the source, and the last is essentially arbitrary. If one is to be arbitrary then, it is believed better to be frankly so in this case and express the gloss in the most convenient fashion, i.e. directly in terms of the reading of the circle, the latter being divided in ordinary degrees.

The worst that can be said of such a scale is that it is at least as rational as the Fahrenheit thermometric scale, and the best that it is practical, convenient, direct-reading and involves no manufacturing difficulties. On the scale chosen a very low gloss, such as that of blotting paper, reads about " 20 degrees," while a high finish-glossy Azo or Solio-comes in the neighborhood of 50. As determinations may be made to the tenth of a degree we may distinguish between these limits a possible 300 gradations of gloss. Ordinary magazine paper runs between 25 and 40 degrees and the stock on which the Optical Society Journal is published averages about 30 . It is hardly necessary to add that all the instruments will be set so that they read the same, with a small calibration correction to be used for accurate work. If one wishes to express gloss in per cent as explained above it is only necessary to make settings at each of the two possible points on the scale, e.g. at 34 degrees, say, and 86 degrees, and take the cosine of the difference.

The external features of the new Glarimeter are shown in the figure although certain improvements which add to the convenience of operation have been incorporated since this photograph was taken. It makes a fairly rugged, compact, portable instrument with which gloss determinations may be quickly made (15 to 20 seconds) without any darkening of the room. It is accordingly

${ }^{4}$ Dr. Nutting in a criticism, Electrical World, 63, 1156 (1914), of my original paper suggests a scale which may be considered absolute but it is doubtful wisdom to apply it here. See also Electrical World, 64, 35 and 717 (1914). 
an essentially practical instrument equally suitable for mill or laboratory use. Nor is its application limited to papers, for it may be used with any surface which does not.show.smetallic reflection: Indeed, in somewhat different form it might prove useful in connection with the finishing of fine furniture or automobiles or in determining the gloss of paints, textiles, walls, etc.

The limitations of the polarization method of paper gloss measurement may be considered for a moment in closing. While it gives a direct measure of the "optical smoothness" or glare-producing property it does not primarily"measure the "mechanical smooth-

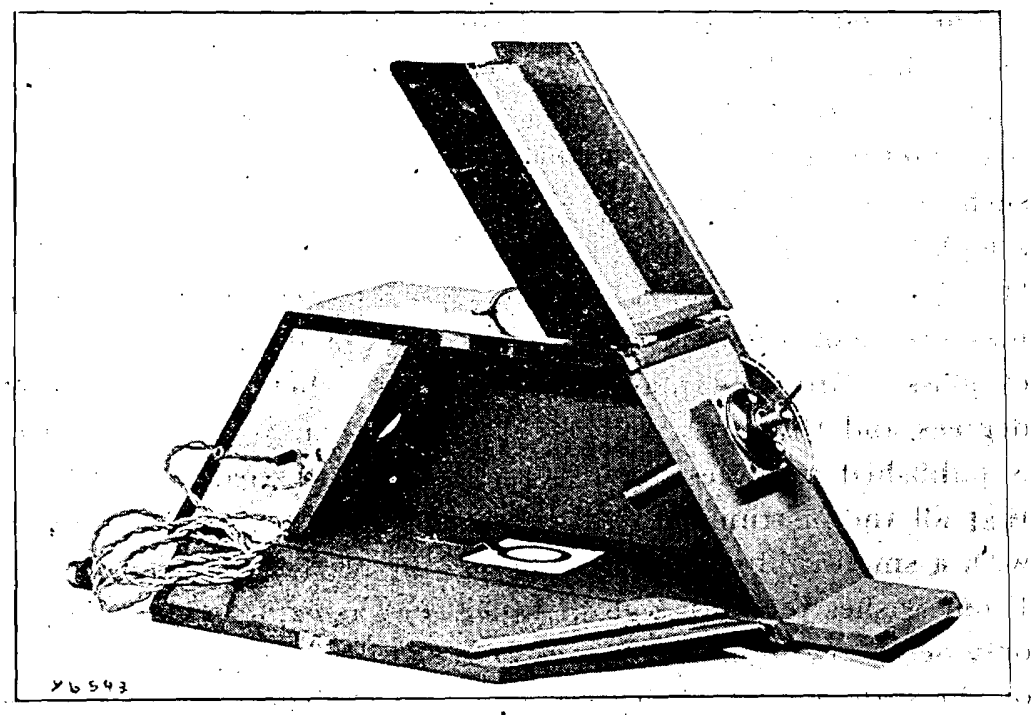

One Form of Glarimeter

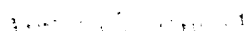

ness" or quality which determines the reproduction of half-tones, and it might at first sight be thought that this would seriously limit its use. Such, however, is not found to be the case. The calendering process which gives the finish to the surface.increases at the same time the optical and mechanical smoothness and in sensibly the same proportion; the measure of one then serves to determine the other as well. It is true that one might imagine exceptions to this-e.g. a smooth-finished paper which has been 
punctured with fine pin-holes: this would still show a high gloss but would be, on the burr side at least, mechanically roughbut such as a rule are highly artificial and never met with in practice. Experience shows that, for any given type of paper, the glarimeter serves every purpose for testing and controlling the uniformity of the calendering process.

When we have under consideration at one time, however, radically different sorts of paper surfaces we cannot generalize so easily. To assume that glarimeter readings alone would serve as an exact measure of the half-tone possibilities of any paper, without reference to the character of its finish, coated or uncoated, etc. would be obviously ridiculous. "This consideration applies particularly to colored papers, where a darkening of shade increases the gloss reading. That this must be so is readily seen by remembering that darkening reduces the amount of diffusely reflected light while it alters only in a minor degree the specularly reflected portion: experiments with solio paper of various shades show this effect very well. It may be said, however, that only in special cases does this affect the usefulness of the instrument. It is found in practice that good settings on colored papers are obtainable when a piece of colored glass is inserted in the eyepiece, and while this should, theoretically, be of the color of the paper, a light red stained glass works very well in practice with a variety of colors.

Physical Laboratory,

UNIVERSITY OF WISCONSIN.

Feb. 7, 1921. 